\title{
Diseño institucional y buen gobierno: avances y reformas pendientes
}

\author{
Ildefonso Hernández Aguado \\ Universidad Miguel Hernández y Ciberesp
}

\section{Resumen}

España carece de una acción política sostenida y estratégica para mejorar la salud de la población más allá del mantenimiento de los servicios sanitarios. El buen gobierno es un requisito para mejorar la política de salud y el diseño del Sistema Nacional de Salud. En este artículo se seleccionan cinco áreas relacionadas con el buen gobierno: priorización, política de salud integral, liderazgo, agencia nacional de salud y entorno cultural e intelectual. Tras analizar las propuestas de diversos autores e instituciones, se seleccionan las acciones que, de acuerdo al contexto actual, podrían implantarse a fin de avanzar en la agenda del buen gobierno.

Palabras clave: buen gobierno, salud, salud pública.

Clasificación JEL: H110, I180.

\begin{abstract}
Spain lacks a sustained and strategic political action to improve the health of the population beyond health services. Good governance is a prerequisite for changing the health policy and the design of the National Health System in the right direction. In this article five areas related to good governance are selected: prioritization, comprehensive health policy, leadership, national health agency, and cultural and intellectual environment. After analysing the available proposals, the actions that according to the current context could be implemented in order to advance the agenda of good governance are examined.
\end{abstract}

Keywords: governance, health, public health.

JEL classification: H11, 1180 .

\section{Introducción}

La salud está ausente del discurso político al más alto nivel en España. No es fácil saber los motivos de esta omisión que es casi una actitud secular de los máximos responsables políticos. Quizá el buen estado de salud de la población y el relativo buen funcionamiento del Sistema Nacional de Salud puede ser parte de la explicación. El Atlas de la salud poblacional en las regiones de la Unión Europea muestra que España ocupa un lugar muy destacado cuando se describen los resultados en salud tanto si se examinan indicadores compuestos o individuales (Santana et al., 2017). Igualmente, los índices sobre la calidad del funcionamiento de los servicios sanitarios sitúan a España entre los países privilegiados. Esta magnífica posición se repite con escasa variación si se emplean aproximaciones metodológicas distintas en la construcción de índices sobre la calidad y el acceso a los servicios sanitarios. Por 
ejemplo, una reciente publicación de la revista Lancet que analiza más de 190 países, otorga a España una puntuación muy alta, si bien inferior a un análisis previo (GBD 2015 Healthcare Access and Quality Collaborators 2017). Si lo que se revisa son los indicadores individuales sobre resultados en salud o sobre calidad de la atención sanitaria y cobertura (OCDE, 2017), España sigue destacando entre los países privilegiados del mundo, especialmente por su esperanza de vida al nacer y a los 65 años, con tan solo algunas cuestiones que merecen mencionarse en la vertiente negativa tales como la alta frecuencia de diabetes o de depresión y el bajo peso al nacimiento con un cambio negativo, de este último indicador, muy pronunciado desde 1990 . Lo mismo ocurre con los indicadores individuales de calidad de atención sanitaria y cobertura, que son positivos, aunque en este caso se detecta un espacio notorio de mejora. Este cuadro tan positivo resulta paradójico, al menos aparentemente, si atendemos al análisis de las causas de los problemas de salud que sitúan a España en un lugar mucho menos favorable y en algún caso claramente desfavorable. El Atlas antes mencionado construye un índice sobre determinantes de salud que comprende: condiciones económicas, protección social y seguridad; educación; cambio demográfico; estilo de vida y conductas relacionadas con la salud; contaminación; condiciones medioambientales (vivienda, saneamiento); seguridad vial; recursos y gasto sanitario; y, funcionamiento del sistema de salud. En este caso, España ocupa una posición intermedia baja en la Unión Europea. Lo mismo ocurre si atendemos a los distintos factores de riesgo y conductas relacionadas con la salud, pues en muy pocos casos España muestra una buena situación. Algunos medios de comunicación expresan la paradoja apuntando a estas conductas relacionadas con la salud: «They drink, they smoke, so why are the Spanish living so long?» (The Times, 2018); o, directamente sugieren posibles explicaciones: «Fruit, veg and family life-Why Spaniards are living longer» (The Guardian, 2018). No es infrecuente comprobar esta paradoja, por ejemplo, hay encuestas de salud en comunidades autónomas en España que han mostrado un perfil de riesgo cardiovascular muy alto que sin embargo no se traducen en morbilidad y mortalidad asociada a medio plazo. En la interpretación de estos datos, debe considerarse que el conocimiento disponible indicó hace ya algún tiempo que las variables conocidas del riesgo cardiovascular explican solo parte del riesgo, por ejemplo, en los países del sur de Europa personas con igual perfil de riesgo que otras en el centro o norte de Europa muestran menor frecuencia de enfermedad (Van del Hoogen et al., 2000). No debemos olvidar por otra parte el alcance de nuestra ignorancia en predecir enfermedad.

Paradojas aparte, y con cierta cautela, se puede decir que algunos indicadores tales como el bajo nivel educativo, el bajo peso al nacer o la alta frecuencia de obesidad infantil en España se pueden asociar a perspectivas de salud negativas en el futuro. Esto también ocurre con otros condicionantes sociales en los que España muestra un perfil preocupante, tal como la pobreza infantil que deja marcas indelebles y que a largo plazo dañan a salud (Rajmil et al., 2018). El hecho de que España sea líder europeo en esperanza de vida o que partamos de una situación relativamente favorable en cuanto a los servicios sanitarios no debería ser pretexto para que la salud 
no ocupe una posición relevante en la agenda política pues hay motivos sobrados para ello, tal como más abajo se explica. Por lo que respecta a la política de salud, puede afirmarse que en España no es identificable una acción política sostenida y estratégica para mejorar la salud de la población más allá de los servicios sanitarios. Aunque es cierto que se han aprovechado las escasas ventanas de oportunidad política para introducir algún cambio decisivo, no hay una inercia positiva que permita esperar que la administración sanitaria, ya sea estatal o autonómica, vaya tejiendo un armazón de normas y actuaciones con vocación de futuro sobre las que avanzar de forma sostenida y perseverante en la protección de la salud. La ley estatal y las autonómicas de salud pública, las leyes de prevención del tabaquismo o el reciente proyecto de ley andaluz para la promoción de una vida saludable son iniciativas tan alentadoras como aisladas. A las leyes de prevención del tabaquismo les ha faltado continuidad, tanto mediante nuevas normas como en actuaciones de refuerzo e inspección y, como consecuencia, estamos en situación de riesgo ante los frecuentes incumplimientos. Las leyes de salud pública han carecido de desarrollo y cumplimiento por cuestiones de mal gobierno. El hecho de que España no cuente con una verdadera política de salud frustra las posibilidades de ganar salud y de contribuir a la sostenibilidad de los servicios sanitarios.

Por lo que respecta al Sistema Nacional de Salud, hay una coincidencia generalizada en que es un buen servicio público pero que afronta graves problemas de sostenibilidad. Vicente Ortún lo dice con claridad: «España tiene un buen sistema sanitario, pero con mal pronóstico salvo que se enderecen algunas tendencias» (Ortún, 2009). Sobre la política y la gestión de los cuidados sanitarios se han realizado innumerables estudios y propuestas. De hecho, se cuenta con análisis suficientemente exhaustivos tanto de sus defectos como de las posibles soluciones como para que fuera más sencillo propiciar cambios en la buena dirección, más aún cuando muchas de las propuestas se han mostrado efectivas en diversos países.

Usualmente se pone todo el foco en los servicios sanitarios asistenciales mientras hay un olvido sistemático de la política de salud y de las actuaciones en salud pública. Pero esa atención casi exclusiva a los servicios asistenciales y sus avatares no ha servido para enderezar sus problemas reiteradamente descritos. Ambas cuestiones, el desinterés por una política de salud digna de tal nombre y la inacción sobre el Sistema Nacional de Salud son consecuencia, entre otros, del mal gobierno y de la baja calidad del diseño institucional. Así se deduce de la mayoría de las propuestas publicadas para mejorar la respuesta social a los retos relacionados con la salud. Proposiciones que avisan de que los importantes problemas de fondo del Sistema Nacional de Salud superan las capacidades actuales de mera administración-gestión, por buena que sea localmente, y que el buen gobierno es una condición previa e indispensable (Freire, 2011). A principios de siglo, al referirse a la generación de políticas públicas saludables, Joan Subirats indicaba que la calidad institucional estaba entre los retos clave de futuro: «España se encuentra en esa, particularmente comprometida, transición entre dos siglos, sin un Estado bien rodado, bien preparado para lo que se avecina y sin una sociedad civil bien enraizada, capaz de asumir 
responsabilidades y estructurar mecanismos de vigilancia y control sobre un espacio público muy frágil. Es ahora cuando nuestro hándicap histórico de instituciones públicas usadas con fines privados y actores sociales débiles, dependientes y con pocos recursos autónomos, puede pasar factura de forma grave» (Subirats, 2001). Desde entonces son muchos los autores que coinciden en que el buen gobierno y la calidad institucional son un paso ineludible para lograr que la respuesta de la sociedad española a los retos de salud sea de alta calidad y sostenible. José Ramón Repullo en su minucioso análisis sobre la sostenibilidad de las prestaciones sanitarias públicas termina escribiendo: «El Sistema Nacional de Salud es sostenible; depende de nosotros, y el futuro no está escrito. Corresponde a la generación de españoles políticamente activos de hoy, el garantizar que legaremos a nuestros hijos y nietos una sanidad pública extensa, amplia, eficiente y de calidad. Y a un coste asumible por la economía. Para ello se requieren reformas estructurales; buen gobierno es la clave para desbloquear esta trayectoria. Y la clara conciencia de que la cohesión y el capital social de los españoles está inevitablemente vinculado a cómo resolvamos este desafío de hacer sostenible nuestro Sistema Nacional de Salud: una adecuada protección de la salud es condición necesaria, aunque no suficiente para construir sociedades decentes. Esto es lo que en la segunda década del siglo XXI nos estamos jugando» (Repullo, 2012a).

En este artículo revisaremos las propuestas de los distintos autores que se han ocupado del asunto, aunque circunscribiendo el enfoque al nivel macro, es decir a los problemas de carácter sistémico de la política de salud. En segundo lugar, abordaré las acciones que cabría considerar para avanzar en la implantación de las medidas propuestas que más consenso aglutinan. En ambos casos adoptaré una perspectiva integral en la que asumo que la gobernanza de los sistemas sanitarios es una parte más de la política de salud. No se pretende obviar aquí que el buen gobierno en salud es una parte más de la calidad de gobierno general tal como más arriba señalaba Subirats, es más, se tiene muy en cuenta lo apuntado por Meneu y Ortún al respecto: «Parece ya ingenuo pretender disociar las peripecias del gobierno sanitario del buen gobierno general de lo público. La caracterización del gobierno -sanitario y no sanitario- en España, el análisis de su impacto en las políticas, la gestión de las organizaciones y las prácticas institucionales, permiten elaborar una agenda tan ambiciosa como factible de las tareas pendientes que los profesionales -en sentido muy amplio- y los responsables sociales debemos acometer con el apoyo ciudadano» (Meneu y Ortún, 2014). Por ello lo que se pretende es identificar una agenda de propuestas convenientes y examinar qué acciones podrían implantarse para desarrollar esa agenda.

\section{Las propuestas para la mejora del buen gobierno y la calidad institucional}

El documento «Sistema Nacional de Salud: diagnóstico y propuestas de avance» de la Asociación de Economía de la Salud (AES) contiene abundantes propuestas 
acerca de del buen gobierno de la sanidad producto del trabajo de numerosos autores y colaboradores con amplia experiencia previa (AES, 2014). Remito al lector interesado a ese excelente trabajo para la explicación del concepto de buen gobierno y sus principios generales que se describen acompañados de referencias bibliográficas oportunas. El cuarto y último capítulo del documento de AES se dedica en exclusiva al buen gobierno de la sanidad. Su primera propuesta se dedica al procedimiento de priorización de políticas sanitarias señalando la necesidad de que sea más participativo y transparente para lo que se pueden usar ejemplos como el del Mandato del gobierno británico a su National Health Service. Esta recomendación sobre necesidad de priorizar y de hacerlo con metodología adecuada, pues un modelo no priorizado no es sostenible, concita un amplio consenso. El que carezcamos de estimaciones adecuadas y comparables de los efectos sanitarios, económicos y sociales de muchas intervenciones (Meneu, 2016), justifica aún más que se acepte que la priorización y la gobernanza son un todo indisociable (Callejón, 2017). De hecho, parte del buen gobierno descansa en el papel de la medición para la priorización y para la rendición de cuentas.

\subsection{Priorización en la política de salud}

Centrándonos en priorización, hay suficientes ideas bien trabajadas en el ámbito circunscrito de los sistemas de salud que están listas para ser aplicadas si se alcanzan las condiciones del entorno político y social para ello (Peiró, 2011) (Del Llano, 2018). Menos trabajados están los procedimientos para la priorización de la política de salud, por ello es oportuno acudir a las buenas prácticas desarrolladas en algunos países como Canadá (González López-Valcárcel, 2018). Al respecto, la experiencia sueca merece atención porque prueba que es posible priorizar áreas estratégicas de ganancia en salud, cuantificar las ganancias esperadas valorando su coste oportunidad y monitorizar el funcionamiento de la política de salud establecida (Linell, 2013). Todo ello se hizo por acuerdo unánime del Parlamento sueco y se plasmó en una ley de salud pública, que indicaba las prioridades y las políticas necesarias. La priorización sueca se basa en los condicionantes de salud y tiene por objetivo nuclear crear las condiciones en la sociedad para una buena salud en términos de igualdad para toda la población. Cuando aquí en España se reunió a expertos en distintos ámbitos para establecer procedimientos de priorización en salud, las prioridades expresadas espontáneamente se acercan a las establecidas en Suecia (Hernández-Aguado, 2016). Lo mismo ocurre con las prioridades expresadas por la Sociedad Española de Salud Pública y Administración Sanitaria (SESPAS, 2015). Esta observación va en la línea de lo apuntado por Ortún «la medición correcta de los valores y preferencias sociales también se puede hacer-sin ciencia-, por medio de la participación responsable de la ciudadanía: todos nosotros, no solo el segmento de beneficiarios, cuando se trata de establecer prioridades para la asignación de recursos financiados con fondos públicos» (Ortún, 2018). La participación, muy nombrada como 
requisito para el buen gobierno, se considera esencial en el futuro de la priorización al igual que también se considera imprescindible atender al entorno ideológico como determinantes del establecimiento de la agenda política y por tanto de la priorización en relación a la atribución de responsabilidad al Estado, el mercado, la familia o el individuo, como también al entorno social en lo referente a los sistemas de valores y creencias más allá de la política (Kickbusch, 2013).

En resumen, el establecimiento de una estrategia nacional de salud basada en las prioridades y necesidades nacionales es un indicador básico incluido en el conjunto de instrumentos de gobernanza de los sistemas de salud y debe integrar cualquier agenda de buen gobierno (World Healh Organization, 2008).

\subsection{Una política integral de salud}

Un segundo ingrediente destacado de los documentos revisados sobre buen gobierno es la necesidad de un abordaje exhaustivo a los problemas de salud, diseñando una política integral. Cada día hay una mayor percepción sobre el papel que juega la salud en alcanzar otras metas sociales benefactoras. Al mismo tiempo la salud es considerada como resultado de las relaciones complejas y dinámicas generadas por numerosos condicionantes en diferentes niveles de gobierno. Los sistemas de salud por sí mismos no pueden resolver de una forma completa y sustancial las causas multidimensionales y sistémicas que están en el origen de los principales problemas de salud (Kickbusch, 2013). La iniciativa de Finlandia sobre Salud en Todas las Políticas, producto de su dilatada trayectoria en implantar políticas saludables para abordar sus problemas de salud más graves, contribuyó a poner de nuevo en la agenda política la necesidad de pensar en políticas de salud y no solo en políticas de servicios sanitarios (Leppo, 2013). Aunque los orígenes de las propuestas sobre políticas saludables son antiguos, la iniciativa formal se puede datar a la Carta de Ottawa. Desde entonces, la salud pública persevera en idear nuevas aproximaciones, denominaciones más atractivas o nuevas estrategias para situar la política de salud en la agenda política, al fin y al cabo, una de las aspiraciones casi seculares de la salud pública, que se desprende de su meta esencial, es garantizar las condiciones en las que las personas puedan tener buena salud. Por lo general, los países con alta calidad democrática y reconocidos por su buen gobierno suele ser aquellos que disponen de una política de salud explícita y bien articulada. En España, la Ley General de Salud Pública supuso un paso en esa dirección, dispuso la elaboración de una estrategia estatal de salud pública que debe ser evaluada periódicamente. Lamentablemente esta ley ya fue cercenada antes de ser remitida al Parlamento y, peor aún, sus disposiciones siguen sin cumplirse siete años después de la promulgación, algo no inusual en España (Hernández-Aguado, 2014 ) (Meneu, 2009). El documento de AES antes mencionado recomienda que se llegue a un pacto sobre la salud apoyado en el desarrollo de Ley General de Salud Pública (Ley 33/2011), que integrara la prevención, la protección y la promoción de la Salud en todas las políticas públicas (AES, 2014). 
En ello coinciden Repullo y Freire, cuando indican en sus recomendaciones de buen gobierno que la Ley General de Salud Pública debe emplearse para asumir el reto de la regulación y gestión de programas de salud pública, y su articulación con las políticas europeas e internacionales, así como invertir los recursos necesarios en la creación y la dotación técnica de la Autoridad Sanitaria Estatal (Repullo, 2016). Igualmente, las propuestas de Oliva et al. para las reformas del Sistema Nacional de Salud consideran que la salud pública, a fin de garantizar que las personas vivan en condiciones saludables, debe trascender en su alcance el ámbito sanitario e identificar y actuar sobre los condicionantes de la salud tales como la atención social, la educación, el mercado laboral, el urbanismo o las políticas, entre otras, en los que se generan o transmiten desigualdades sociales y de salud (Oliva, 2013). También Cabases cree que la salud pública es una línea básica del buen gobierno de los servicios de salud y entiende que la salud debe ser el hilo conductor del sistema, revitalizando la salud pública e impulsando la prevención, la protección y la promoción de la salud, haciendo de la buena salud, y de la calidad de vida ligada a ella, una de las grandes prioridades de todas las políticas públicas (Cabases Hita, 2013). Como en otros asuntos, Ortún sintetiza la cuestión con claridad en el título de un comentario en Gaceta Sanitaria: «A la política rogando y con el mazo dando (en los servicios sanitarios)» proponiéndolo como lema de la necesaria conciliación entre las acciones individuales y las colectivas sobre la salud (Ortún, 2007).

Sería desafortunado que se considerase la necesidad de una política de salud integral como una cuestión complementaria para facilitar la sostenibilidad del Sistema Nacional de Salud, cuando lo lógico es que esta fuera una cuestión previa e imprescindible. Esta perspectiva es si cabe más necesaria en la situación actual derivada de la globalización que ha cambiado el equilibrio de poder entre los estados y los mercados y se necesita una adaptación institucional. Se requiere un conjunto de políticas sinérgicas que implican a un amplio rango de actores para tratar los problemas de salud pública emergentes y actuales; es más, un país individualmente no tiene la capacidad ni los instrumentos adecuados para resolver adecuadamente los problemas multidimensionales que suponen los retos actuales de salud. En esta línea de salud en todas las políticas se habla de enfoques del conjunto del gobierno y del conjunto de la sociedad, «whole-of-government and whole-of-society approaches» y se considera que es necesario cambiar las principales prácticas institucionales reuniendo diversos actores, redes y coaliciones, incluyendo el gobierno, la comunidad y representantes empresariales. Con ello se pretende establecer políticas sinérgicas implicando a un amplio rango de actores para tratar los problemas de salud pública emergentes y actuales. Son los países anglófonos los que más han desarrollado esta visión acuñando denominaciones como «joined-up government» en Reino Unido, «horizontal government» o «horizontal management» en Canadá, «integrated government» en Nueva Zelanda, «networked government» en Estados Unidos y «whole of government» en Australia y Escocia (Kickbusch, 2013). Este asunto subyacía también en la intención del informe sobre la medición del progreso social y funcionamiento económico comisionado por Nicolás Sarkozy (Stiglitz, 2009). 
La idea de acuñar denominaciones atractivas e incluso diseñar procedimientos para facilitar las acciones sinérgicas de los gobiernos es plausible si bien debe considerarse con precaución la noción más o menos implícita de gobernanza compartida por la que a veces se pretende invitar a la mesa de toma de decisiones a los diversos actores, cuando hay asimetrías de poder obvias. Este enfoque no debería obviar que algunos actores interesados ya están actuando en política desde hace tiempo, en el caso de la salud pública ya se han descrito modelos de gobernanza con la participación de la industria que ha desembocado en la captura de la política de salud y un freno a la regulación (Miller, 2010). Por otra parte, y tal como describe Subirats, aunque haya una amplia pluralidad de actores en política de salud con aparente capacidad de influir en el proceso de elaboración de políticas, no debe olvidarse la clara desigualdad que hay en el escenario público (Subirats, 2001). La amplia disponibilidad de recursos de las empresas relacionadas con la salud, causa un déficit democrático, no solo por su actuación directa sino porque logran que la mayor parte de actores esté alineado con sus intereses. En cualquier caso, no cabe duda de que hay que encontrar soluciones para un entorno social y económico en el que la distribución del poder ha cambiado sustancialmente y en el que el papel de las corporaciones trasnacionales como determinantes de la salud es creciente y de gran magnitud (Hastings, 2012) (Moodie, 2013).

En España, el reto al que nos enfrentamos estriba en cambiar radicalmente la actual inacción y desgobierno o lo que es una política de salud convertida en una serie inconexa de propuestas de gabinete. Como han descrito Repullo e Infante, en el Ministerio de Sanidad sucede lo que se ha denominado «gabinetización» de la política: «es decir, al peso cada vez mayor de los gabinetes del Ministro, los Secretarios Generales y los Subsecretarios (incluido el gabinete de prensa) en el diseño y el control de las políticas en detrimento de las unidades de técnicas de línea (es decir, de las Direcciones Generales) con el riesgo de subjetivismo y arbitrariedad que ello a menudo implica» (Repullo, 2013). Esto es posiblemente consecuencia de algunos de los vicios políticos exacerbados desde la transición, en palabras de Meneu y Ortún: «Entre estos destacan la vinculación "natural" de su dirección a la voluntad de la articulación política triunfante, sin que ello suponga necesariamente cambios en las políticas sanitarias, pero sí relevos en los directivos en función de sus lealtades y confianzas, y la consiguiente "despolitización" (que no "despartisanización") de la acción política, con crecientes componentes de "bandería" y enfrentamiento espectacular» (Meneu y Ortún, 2011).

Es esperable que las prioridades de salud que puedan establecerse en España se aproximen a las de algunos países de la Unión Europea más arriba citadas y también tengan bastante que ver con las recomendaciones emitidas por la Comisión sobre los Determinantes Sociales de la Salud de la Organización Mundial de la Salud (Comisión sobre los Determinantes Sociales de la Salud, 2009). De hecho, las propuestas de políticas de salud del informe de AES, si exceptuamos la timidez en el uso de la regulación, van en esa dirección (AES, 2014). Por ello no hay duda de que es urgente acordar una política de salud integral que establezca metas y estrategias apropiadas 
de acuerdo a los problemas y retos de la salud y guiada por los principios generales de buen gobierno.

\subsection{Liderazgo}

El camino hacia un buen gobierno de la salud que abarque las características indispensable requiere liderazgo, se trata de alcanzar los atributos que son específicos del buen gobierno que AES enumera citando a la Comisión de Buen Gobierno Sanitario del Consejo de Europa (AES 2014): rendición de cuentas, transparencia, responsabilidad, participación, eficiencia, calidad, seguridad, y dar respuesta a las necesidades de los ciudadanos; y en aspectos éticos: una cultura de integridad, códigos de conducta, atención a los conflictos de intereses y prevención del fraude y la corrupción. Ese liderazgo puede englobar la función de rectoría (stewardship), que busca reactivar el liderazgo de los ministerios de salud y su capacidad de establecer y desarrollar estrategias que orienten al sector y sistema sanitario hacia ganancias de salud, y también puede incluir la función de gobernanza, como aquella que persigue restaurar mecanismos y herramientas de regulación, jerarquía, organización o incentivación para hacer posible la implementación de las políticas. La Organización Mundial de la Salud (OMS) en el informe de salud del mundo en el año 2000, asume que la rectoría en materia de salud es la esencia misma del buen gobierno y además de definir la visión y la dirección de la política de salud, como antes se menciona, debe ejercer influencia por medio de la reglamentación y la defensa de la causa (OMS, 2000). Ya en ese ese año, la OMS consideraba que era una miopía rectora de los ministerios de salud el hecho haber perdido de vista del objetivo de la política de salud dirigida al conjunto de la población y el no reconocer que todo el gobierno debe tener función rectora en salud. Igualmente recomendaba que las autoridades en salud entablasen coaliciones con otros actores para la defensa de la salud y para facilitar los cambios sin basarse únicamente en la regulación. La rectoría, es considera por Repullo e Infante como la adecuada combinación de legislación adecuada, información transparente, normas técnicas oportunas y fondos complementarios de asignación finalista y condicionada que influyeran en el comportamiento de los distintos actores (Repullo, 2013), lo que desde luego sería un buen comienzo en estos momentos. Entre las magníficas recomendaciones del Consejo Asesor sobre el Buen Gobierno de la Sanidad Pública Vasca, se incluye la siguiente: fortalecer, si cabe, el papel que corresponde al Consejero de Sanidad y al Departamento de dirección política, orientación estratégica y fijación de objetivos generales para el gobierno del conjunto de la sanidad pública vasca, diferenciando este rol del que está delegado en el Consejo de Administración de Osakidetza/SVS, en sus órganos directivos y en organizaciones (comarcas, hospitales, etc.) (Consejo Asesor sobre el Buen Gobierno de la Sanidad Pública Vasca, 2012). Creemos que el liderazgo, junto con los ingredientes mencionados sobre rectoría, son esenciales para un buen gobierno y para favorecer decisiones políticas saludables. 
En cuestiones relativas a las decisiones de políticas públicas, Subirats y Dente analizan el conocimiento hasta ahora disponible contrastando los diversos enfoques (Dente, 2014a). Del contraste de posiciones de diversos autores que describen, interesa destacar que mientras unos asumen que el poder político está bastante distribuido en las democracias modernas en lugar de estar concentrado, otros señalan que no solo importa saber quién influye en las decisiones, también cabe revisar las formas sutiles de actuación como por ejemplo aquellas destinadas a impedir que determinadas cuestiones entren en el proceso decisional. De hecho, muchas «no decisiones» son «decisiones de no decidir» en línea con la definición de policy que propone Dye: «todo lo que los gobiernos deciden hacer o no hacer». En política de salud la inacción es habitual, ahora mismo es patente que hay un problema con el juego (las apuestas o similares) en España y sin embargo, ni hay liderazgo político desde la administración para dar respuesta, ni iniciativas concretas más allá de las que han propuesto dos grupos parlamentarios.

Lukes afirma que el mayor poder consiste en la capacidad de modelar las preferencias de los individuos, de modo que sean inducidos a apoyar elecciones que en realidad van contra sus intereses (Dente, 2014b). Callejón et al. resaltan que para que una sociedad se desarrolle, es necesario que sus instituciones hagan individualmente atractivo lo que sea socialmente conveniente (Callejón, 2018). Pero también señalan que las preferencias son lábiles. Es por ello que es imprescindible la intervención de los poderes democráticamente elegidos para debatir sobre las preferencias en la esfera pública. La autoridad sanitaria debe entrar en la arena pública para la defensa de la salud de la población, se trata de facilitar la adopción de políticas de salud, de comunicar las políticas y las líneas estratégicas a la ciudadanía, de rendir cuentas sobre sus efectos. Se trata también de promover el debate parlamentario con la idea de mostrar la integralidad a todo el gobierno y la sociedad de la política de salud, tal como sugieren Repullo y Freire, propiciar debates tipo «estado sanitario de la nación/comunidad autónoma» como una excelente palanca para interesar y fomentar políticas multisectoriales y gubernamentales saludables (Repullo, 2016). La actual agenda de intervenciones de las personas con máxima responsabilidad política de España o de sus comunidades autónomas no se acerca a la función de rectoría, de liderazgo que deberían asumir. Un liderazgo que además debería perfilar las decisiones sobre qué diseño institucional debe tener el Ministerio de Sanidad.

\subsection{Agencia Nacional de Salud}

Si hay algo en el diseño institucional sobre el que hay casi unanimidad es sobre la perentoria necesidad de una agencia de alto nivel técnico de salud capaz de evaluar políticas y tecnologías. En la prensa especializada se ha llegado a hablar de «clamor por un Hispanice» (Barbado, 2014). Se le denomina HispaNice a la semejanza deseada con la agencia británica National Institute for Health and Care Excelence (NICE). 
Hay motivos para usar el NICE como referencia, pues ha sido descrito como ejemplo de buen gobierno. Por ejemplo, el Observatorio Europeo de Políticas y Sistemas de Salud que propone TAPIC (transparencia, rendición de cuentas, participación, integridad y capacidad) como marco de buen gobierno, menciona de forma reiterada en su publicación el papel favorable del NICE en el refuerzo de la gobernanza de los sistemas de salud (Greer, 2016). Por su parte, la segunda recomendación de Repullo y Freire para mejorar el gobierno institucional del Sistema Nacional de Salud a nivel macro es la siguiente: «Creación de la Agencia del Sistema Nacional de Salud con nuevos instrumentos de coordinación operativa: fondos de cohesión, de compensación y de inversión; evaluación de tecnologías y medicamentos (Hispa-NICE); y aplicación de criterio de transparencia reforzada y comparación abierta en todos los sistemas de información de comunidades autónomas, centros y servicios» (Repullo, 2016). Cabe subrayar que los borradores de la Ley General de Salud Pública habían diseñado una agencia que reuniese las capacidades de todo el estado de forma articulada y eficiente para poner lo mejor del país al servicio del conjunto, es decir, de la comunidad autónoma que lo necesitase y del Gobierno de España, así como para actuar a nivel internacional de forma competitiva dadas las capacidades técnicas y científicas disponibles. Las resistencias del Ministerio de Economía opuesto en esos momentos -2010 - a cualquier nueva agencia, junto a las del propio Ministerio de Sanidad obligaron a que lo que era un capítulo completo quedase en un solo artículo de la ley que establece la creación del Centro Estatal de Salud Pública con un alcance, en principio, limitado y pendiente del cumplimiento de la ley.

Las agencias no son una panacea y pueden ser vulnerables a la captura por actores interesados, incluso se ha esgrimido que las acusaciones de captura son una forma deliberada de tratar de que no haya agencias. El NICE, tal como indica el antes citado del Observatorio Europeo de Políticas y Sistemas de Salud, es un ejemplo de buen gobierno que ha afrontado las posibilidades de captura demostrando, como se evidencia en la obra de Carpenter y Moss sobre prevención de captura regulatoria, que es posible proteger las agencias de la captura (Carpenter, 2014).

No hay duda de que una agencia de salud requiere una alta reputación de competencia y eficacia técnica tanto dentro como fuera del gobierno. La cuestión es qué tipo de agencia es necesaria, cuáles son sus funciones, que diseño institucional se articula para garantizar su independencia y, finalmente, como se consigue que sea social y políticamente deseable. Una propuesta que cuenta con bastante consenso es la de AES: «Crear una agencia u observatorio de evaluación de servicios sanitarios y políticas de salud cuyos rasgos distintivos sean la imparcialidad, el rigor científico, la participación y la transparencia. Para asegurar su independencia, los recursos de dicha agencia dependerían de los Presupuestos Generales del Estado y rendiría cuentas directamente al Parlamento, quien aseguraría su existencia durante un tiempo suficientemente prolongado para evaluar su utilidad social» (AES, 2014). Sin embargo, si consideramos la incardinación de la salud y su papel central dentro de las metas generales de bienestar social y de acuerdo al principio de salud en todas las políticas la posibilidad de un instituto nacional para la mejora del bienestar, 
asumiendo las características anteriores, podría ser una opción apropiada (González López-Valcárcel, 2010).

\subsection{El entorno cultural e intelectual}

El entorno intelectual y cultural tienen influencia decisiva en la viabilidad de las acciones para mejorar el buen gobierno. Por ejemplo, un entorno público en el que faltan posiciones autorizadas y acreditadas en materia de salud facilita la captura de políticas, da lugar a aparente equivalencia entre las diversas posiciones y propuestas que facilita los objetivos de agentes interesados. De ahí que algunos actores con influencia en la política de salud prefieran la ausencia de agencias técnico-científicas y de voces autorizadas. La emisión de recomendaciones científica y socialmente fundamentadas en salud pública es precisamente uno de los papeles que debe cumplir una agencia de salud. Tal como describe Cuellar, acerca de la actuación de los Centers for Disease Control and Prevention en diversos problemas de salud a lo largo de su historia, la alta reputación científica y técnica en la sociedad estadounidense contribuyo a evitar la captura de políticas (Cuellar, 2014). A propósito, vale la pena recordar que, en ausencia de agencias científico-técnicas en salud, hay organizaciones de la sociedad civil que pueden emular el papel. Eso es lo que en cierta forma hizo el Comité Nacional de Prevención del Tabaquismo para favorecer la legislación de prevención del tabaquismo pasivo en España (Hernández-Aguado, 2013).

La percepción de los riesgos de mal gobierno es escasa, el entorno social no lo favorece. Muchos de los actores interesados en la política de salud no perciben los conflictos y los potenciales problemas éticos cuando participan en actuaciones relacionadas con la salud. Puede ser que en ocasiones se sea consciente de las contradicciones, pero la impresión es que lo que ocurre es producto del curso natural de los acontecimientos. Por ejemplo, de la misma forma que un médico interno residente no percibe nada inusual cuando en su primera semana de trabajo se celebra una cena de bienvenida financiada por una industria relacionada con la salud, una asociación padres y madres de familia no encuentra inconveniente alguno en adherirse a una campaña de promoción de salud infantil auspiciada por una empresa que comercializa productos insalubres. El proceso y fracaso en la legislación sobre la protección de menores ante el consumo de bebidas alcohólicas, sustituyéndolo por la autorregulación ilustra bien el asunto (Hernández-Aguado, 2018). La Ministra de Sanidad en 2011 presidió la firma de un gran acuerdo social para lograr el «consumo cero» de alcohol entre los menores de edad. A la firma pudieron asociaciones de padres y madres, jóvenes, educadores, ONG, sociedades científicas, productores de bebidas alcohólicas, cerveceros, distribuidores, hosteleros, y medios de comunicación entre otros. El acuerdo tenía por primer objetivo evitar toda comunicación, información, publicidad o promoción comercial que relacione el consumo de bebidas alcohólicas con el éxito social o sexual, con la mejora del rendimiento físico o que induzca al consumo de alcohol por parte menores de edad. Mientras se firmaba el acuerdo unas 
latas de cerveza incluían la imagen de jugadores de la selección española de fútbol. La siguiente ministra celebró un acuerdo similar con los fabricantes de bebidas espirituosas al tiempo que usaba la ley de presupuestos para facilitar la publicidad de bebidas alcohólicas de graduación inferior a 20 grados donde no se podía vender y consumir. El proceso ha concluido por el momento con la concesión de la Cruz Blanca de la Orden al Mérito del Plan Nacional sobre Drogas a la Federación Española de Bebidas Espirituosas por parte del Ministerio de Sanidad, Servicios Sociales e Igualdad en 2016 (Hernández-Aguado, 2018). Esta baja capacidad para detectar riesgos de mal gobierno también puede percibirse en altos cargos cuando las propuestas de actores interesados son atractivas, que es lo habitual cuando se plantean colaboraciones publico privadas para la promoción de la salud (Hernández-Aguado, 2016). Incluso, en los ámbitos científicos la percepción sobre estas colaboraciones puede ser positiva por la influencia que comentarios y editoriales pueden tener en el entorno científico y que pueden ser producto de captura científica (Zaragoza Gaynor, 2016) (Hernández-Aguado, 2016).

El que la respuesta social a los problemas de salud sea preferentemente de carácter biomédico es en parte consecuencia de cierta captura del entorno por parte de agentes interesados. Hay que recordar que prácticamente toda la prensa especializada en sanidad está financiada por agentes interesados y en sus páginas raramente tienen espacio intervenciones de salud que no sean patentadas. La capacidad de influencia en la esfera pública de las industrias relacionadas con la salud es alta como corroboran las palabras de un responsable de relaciones institucionales de una importante compañía farmacéutica recogidas en el informe sobre el lobby en España: «Una característica de la industria es la calidad y cantidad de información y recursos de que dispone. Una multinacional de cierta magnitud tiene información detallada y puntual sobre todos los procesos de su interés (tanto científicos como de capacidad de prospección, conocimiento de procesos internacionales comparados, funcionamiento de la administración y personas clave, etc.), que está a mucha distancia de la que tienen las administraciones. Esta información le otorga a la industria una enorme capacidad de adelanto a los acontecimientos. En comparación, las administraciones tienen escasos recursos y una información desordenada, de baja calidad, casi grotesca. En estas condiciones la capacidad de influencia indirecta es alta porque se domina el entorno científico, intelectual, formativo, político, etc. y en consecuencia la influencia directa se ejerce solo en casos imprescindibles. La necesidad de influir directamente en la toma de decisiones viene determinada por la ausencia de un campo de juego limpio, de una regulación clara y previsible. Por ello, si es necesario, se ejerce presión con toda la intensidad y en todos los niveles del gobierno al alcance» (Villoria Mendieta, 2014). En ese mismo informe se detallan los actores que juegan un papel relevante en la política de salud y las estrategias de influencia empleadas.

La influencia de la prensa en la esfera pública y en el resto de actores es esencial, ya antes hemos descrito la denominada gabinetización de la política de salud en España más pendiente del dossier de prensa que de cualquier objetivo, aunque este estuviese en el programa del partido que sustenta el gobierno. Según Charron 
et al., tres factores explicarían las diferencias de calidad de gobierno entre países: una gestión pública profesional con una separación estricta entre las carreras de políticos y funcionarios; una descentralización y autonomía en la gestión de los recursos humanos; y, transparencia, entendida como el acceso a la información pública (sin publicidad ni ocultando los malos resultados), y libertad de prensa. La propiedad actual de los medios de comunicación dificulta el acceso a información veraz e influye en la agenda pública condicionando así la agenda política. Un asunto crucial es la definición del problema que da lugar o desencadena la política. Definir un problema no es la simple descripción de una situación que no nos gusta y que se desea cambiar (Subirats, 2000). En el proceso de formulación de políticas la batalla por definir de una determinada forma los problemas es clave porque es la forma en la que se organizan y se da sentido a los asuntos, es decir, se seleccionan algunos aspectos de la realidad y se destacan para promover una determinada definición del problema, una interpretación causal, una evaluación moral y una recomendación de solución determinada (Entman, 2009). Los medios y los actores que puedan influir en el establecimiento de la agenda mediática y el framing (definición) influyen en el contexto cognitivo, no solo dando relevancia a unos problemas y silenciando otros sino definiendo el problema en sí de forma que se anticipa un tipo de solución. En nuestro entorno, los medios generales también contribuyen a anticipar soluciones biomédicas a los problemas de salud, y han contribuido, por ejemplo, a enmarcar las propuestas de regulación para proteger a los menores del alcohol como un atentando a la libre elección. Por último, y respecto al entorno intelectual, es preciso señalar la necesidad de abordar la producción y difusión del conocimiento. La distorsión de la ciencia alcanza niveles preocupantes y contribuye a perfilar percepciones en la esfera pública no favorables a algunas políticas de salud tanto directamente como con la ayuda de los medios de comunicación. La atención al entorno intelectual y cultural es necesaria para cualquier estrategia de buen gobierno y exige a los responsables políticos y a las administraciones trabajar sobre cómo influir y sobre qué definiciones emplear en promocionar sus actuaciones.

\section{Avanzando en la agenda de buen gobierno}

Las acciones para avanzar en la agenda de buen gobierno son, en general, de carácter transversal. Las que a continuación se exponen no son de carácter integral o amplio, son tan solo propuestas concretas que pretenden contribuir a crear un entorno favorable a las decisiones sobre buen gobierno.

La primera, mencionada con mucha frecuencia, es la formación. En la agenda de organismos e instituciones no gubernamentales relacionados con la salud podrían incluirse acciones de influencia al Ministerio de Sanidad, Consumo y Bienestar Social para que el Consejo Nacional de Especialidades en Ciencias de la Salud incluya en todos los programas de formación de los futuros especialistas contenidos sustantivos de buen gobierno (transparencia, independencia, imparcialidad, conflictos de 
intereses, etc.). Igualmente cabe influir en las consejerías de sanidad de las comunidades autónomas para que la formación en buen gobierno sea un mérito destacado en el acceso a los puestos de trabajo. De forma sinérgica debe proponerse un encuentro de entidades de formación de postgrado en ciencias de la salud para que, si es posible en alianza, se preparen currículos de formación en buen gobierno. También es relevante la formación de los funcionarios e incluso más importante aún de los altos cargos de las consejerías, por lo que debe procederse de forma similar a lo más arriba expuesto.

Para que estas acciones de influencia se refuercen, las organizaciones civiles interesadas podrían identificar algún responsable sanitario de alguna comunidad autónoma proclive al buen gobierno y que desee llevar al Consejo Interterritorial del Sistema Nacional de Salud una propuesta de buen gobierno en línea con lo antes planteado.

La aparente intención actual sobre el desarrollo de la Ley General de Salud Pública debe aprovecharse para que aquellos artículos que requieren reglamento, este sea lo más exigente posible en las cuestiones de buen gobierno. Esto ocurre por ejemplo con el artículo 11 sobre colaboración en salud pública e imparcialidad en las actuaciones sanitarias cuyo reglamento podría ser ambicioso asumiendo que muchas recomendaciones influyen en la salud pública y reglar los requisitos de imparcialidad y declaración de interés que afectasen a muchas comisiones de expertos, guías de actuación, etc. El Título II de la Ley sobre la planificación y coordinación de la salud pública es otro ejemplo entre otros muchos de la norma que deben desarrollarse y que, en este caso, podría permitir la redacción de una política de salud integral.

Organizaciones interesadas pueden instar al Ministerio o a las consejerías para establecer normas (órdenes, decretos u otros) que afecten a las recomendaciones sobre salud y sobre atención sanitaria, como son las guías clínicas, para incrementar su imparcialidad y que estableciesen que la colaboración de la administración con otros agentes se guiará por los principios de buen gobierno con exigencias claras al respecto. Esto podría hacerse con la participación de las sociedades científicas, colegios profesionales y otros actores.

Sobre la cuestión de una agencia independiente y dadas las barreras bien descritas por Abellán y Martínez, es preciso centrarse de momento en el diseño, en definir una metodología clara y trasparente que incluya la participación de todos los agentes implicados y que describe su diseño institucional de acuerdo al contexto político español (Abellán Perpiñán, 2016). Es preciso que más allá de las recomendaciones pasemos propuestas articuladas, es decir, a ofrecer un documento con un diseño listo para el uso que puede ser presentado y discutido pergeñando un ámbito apropiado. Organizaciones tan activas como por ejemplo AES podrían, más allá de ofrecer opiniones razonadas y recomendaciones solventes, ofrecer documentos de gobierno listos para uso indicando quién, qué y cómo deben hacerse las cosas.

El Comité Nacional de Prevención del Tabaquismo hizo funciones de agencia para estimular la legislación preventiva. SESPAS, que es una organización que reúne 12 sociedades científicas del ámbito de la salud pública y la administración sanitaria, y otras organizaciones deben articular organismos similares tanto para promover el 
buen gobierno, el Comité Nacional de Buen Gobierno Sanitario, como para acciones concretas de política de salud (nutrición saludable, salud y cambio climático, etc.). La función de estos organismos es influir en el entorno mediático y político y propiciar documentos de base para estimular políticas favorables al buen gobierno y a la salud. Por ejemplo, una de las tareas del Comité Nacional de Buen Gobierno Sanitario consistiría en redactar una propuesta formal de Agencia Nacional de Salud.

Es cierto que muchas de estas acciones requieren del voluntarismo, pero el hecho de que gocemos de una alta esperanza de vida con calidad puede favorecer la participación de jubilados activos cada vez más numerosos y con mayor capacidad.

Algo similar cabría hacer para propiciar la implantación de algunas de las propuestas, tales como la priorización, el liderazgo o el entorno intelectual y cultural. Varias de las acciones nombradas van en esa dirección y podrían adaptarse. El adelgazamiento de las administraciones hace que de momento no sean esperables cambios notables en estas áreas. No cabe más que trabajar desde la sociedad civil para configurar instituciones y acciones en paralelo como las antes mencionadas para que paulatinamente influyan para el cambio. El reto pasa por lograr que haya actores sociales sólidos, independientes y con recursos autónomos. Para ello son necesarias obras y no solo razones.

\section{Referencias bibliográficas}

[1] ABELLÁN PERPIÑÁN, J. M. y MARTÍNEZ PÉREZ, J. E. (2016). «Cómo articular la cooperación en red de los recursos sobre conocimiento y evaluación en salud y servicios sanitarios en España. ¿Hacia un HispaNICE?». Gaceta Sanitaria, 30, Supl. 1, 14-8.

[2] ASOCIACIÓN DE ECONOMÍA DE LA SALUD (AES). (2014). «Sistema Nacional de Salud: diagnóstico y propuestas de avance». AES, 113-130. Recuperado en noviembre 2018 de http://www.aes.es/Publicaciones/SNS_version_completa.pdf.

[3] BARBADO, J. (2014). «Los directivos del sector se unen al clamor por un 'Hispanice'». Redacción Médica, 17 de febrero. Recuperado en noviembre 2018 de https://www. redaccionmedica.com/noticia/los-directivos-del-sector-se-unen-al-clamor-por-un-hispanice-9350.

[4] CABASÉS HITA, J. M. (2013). «El buen gobierno de los servicios de salud». Derecho y Salud, 23, 33-51.

[5] CALLEJÓN, M.; CAMPILLO, C. y ORTÚN, V. (2018). «Deciding on public programs. Prioritisation and governance: an inseparable whole». En J. del Llano y S. Peiró (dirs.), Prioritising or muddling through. Madrid, Springer Healthcare, 1-22. Recuperado en noviembre 2018 de http://www.fgcasal.org/publicaciones/Prioritising-Health-Services-or-Muddling-Through.pdf.

[6] CARPENTER, D. y MOSS, D. (eds.) (2014). «Preventing Regulatory Capture: Special Interest Influence and How to Limit It». The Tobin Project. New York, Cambridge University Press. Recuperado en noviembre 2018 de https://tobinproject.org/sites/ tobinproject.org/files/assets/Introduction\%20from\%20Preventing\%20Regulatory\%20 Capture.pdf. 
[7] CHARRON, N.; DIJKSTRA, L. y LAPUENTE, V. (2015). «Mapping the regional divide in Europe: a measure for assessing quality of government in 206 European regions». Social Indicators Research, 122, 315-346.

[8] COMISIÓN SOBRE LOS DETERMINANTES SOCIALES DE LA SALUD. (2009). «Subsanar las desigualdades en una generación: alcanzar la equidad sanitaria actuando sobre los determinantes sociales de la salud». Ginebra, Organización Mundial de la Salud. Recuperado en noviembre 2018 de http://www.who.int/social_determinants/ thecommission/finalreport/es/.

[9] CUÉLlAR, M. F. (2014). «Coalitions, Autonomy \& Regulatory Bargains in Public Health Law». En D. Carpenter y D. A. Moss (eds.), «Preventing Regulatory Capture: Special Interest Influence and How to Limit It». The Tobin Project. Nueva York, Cambridge University Press, 326-62. Recuperado en noviembre 2018 de https://tobinproject.org/sites/tobinproject.org/files/assets/Introduction\%20from\%20Preventing\%20Regulatory\%20Capture.pdf.

[10] DEL LLANO, J. y PEIRÓ, S. (dirs.). «Prioritising or muddling through». 23-35. Recuperado en noviembre 2018 de http://www.fgcasal.org/publicaciones/Prioritising-Health-Services-or-Muddling-Through.pdf.

[11] DENTE, B. y SUBIRATS, J. (2014a). Decisiones públicas. Análisis y estudio de los procesos de decisión en políticas públicas. Barcelona, Planeta, 15-33.

[12] DENTE, B. y SUBIRATS, J. (2014b). Decisiones públicas. Análisis y estudio de los procesos de decisión en políticas públicas. Barcelona, Planeta, 29.

[13] DYE, T. R. (1987). Understanding Public Policy. Chicago, Englewood Cliffs, Prentice Hall.

[14] ENTMAN, R. M.; MATTHES, J. y PELLICANO, L. (2009). «Nature, Sources, and Effects of News Framing». En K. Wahl-Jorgensen y T. Hanitzsch (eds.), The Handbook of Journalism Studies. New York, Routledge, 175-190.

[15] GBD 2015 Healthcare Access and Quality Collaborators (2017). «Healthcare Access and Quality Index based on mortality from causes amenable to personal health care in 195 countries and territories, 1990-2015: a novel analysis from the Global Burden of Disease Study 2015». Lancet, 390,231-266.

[16] GONZÁLEZ LÓPEZ-VALCÁRCEL, B. (2018). «Prioritisation in Public Health: Aims, Methods, Problems and Practical Experiences». En J. del Llano y S. Peiró (dirs.), Prioritising or muddling through, 23-35. Recuperado en noviembre 2018 de http://www. fgcasal.org/publicaciones/Prioritising-Health-Services-or-Muddling-Through.pdf.

[17] GONZÁLEZ LÓPEZ-VALCÁRCEL, B. y ORTÚN, V. (2010). «Putting health in all welfare policies: is it warranted? A Southern European perspective». Journal of Epidemiology and Community Health, 64, 497-9.

[18] CONSEJO ASESOR SOBRE EL CÓDIGO DE BUEN GOBIERNO DE LA SANIDAD PÚBLICA VASCA. (2011). Recomendaciones. Consejería de Salud y Consumo. http:// www.euskadi.eus/contenidos/informacion/cbil_buengobierno/es_cbil/adjuntos/Buengobiernomarzo.pdf.

[19] GREER, S. L.; WISMAR, M. y FIGUERAS, J. (eds.) (2016). Strengthening Health System Governance. Better policies stronger performance. Maidenhead (UK), McGraw Hill, Open University Press.

[20] HASTINGS, G. (2012). «Why corporate power is a public health priority». British Medical Journal, 345:e5124. 
[21] HERNÁNDEZ-AGUADO, I. (2013). «The tobacco ban in Spain: how it happened, a vision from inside the government». Journal Epidemiology and Community Health, 67, 542-3.

[22] HERNÁNDEZ-AGUADO, I. (2014). «El gobierno de las crisis de salud pública». El Mundo, 14/10/2014. Recuperando en noviembre 2018 en https://www.elmundo.es/salud/2014/10/14/543c2c78ca47410e258b456f.html.

[23] HERNÁNDEZ AGUADO, I. (coord.) (2016). Definición de prioridades en las políticas de salud. Barcelona, Fundación Dr. Antonio Esteve, 36.

[24] HERNÁNDEZ-AGUADO, I. y ZARAGOZA, G. A. (2016). «Support of public-private partnerships in health promotion and conflicts of interest». British Medical Journal Open, 18, 6, 4, e009342

[25] HERNÁNDEZ AGUADO, I. y VILLALBÍ, J. R. (2018). «Public-private interaction with the alcohol industry and failures in the regulation of alcohol in Spain». En UK Health Forum (eds.), Public health and the food and drinks industry: The governance and ethics of interaction. Lessons from research, policy and practice. London, UKHF, 96-101. Recuperado en noviembre 2018 de https://www.idrc.ca/sites/default/files/sp/ Documents\%20EN/ukhf-casebook-jan18.pdf.

[26] KICKBUSCH, I. y BEHRENDT, T. (2013). Implementing a Health 2020 vision: governance for health in the 21st century. Making it happen. Copenhagen, WHO Regional Office for Europe. Recuperado en noviembre 2018 de http://www.euro.who.int/_data/ assets/pdf_file/0018/215820/Implementing-a-Health-2020-Vision-Governance-forHealth-in-the-21st-Century-Eng.pdf.

[27] LEPPO, K.; OLLILA, E.; PEÑA, S.; WISMAR, M. y COOK, S. (2013). Health in all policies: seizing opportunities, implementingpolicies. Helsinki, Ministry of Social Affairs and Health. Recuperado en noviembre 2018 en http://www.euro.who.int/data/ assets/pdf_file/0007/188809/Health-in-All-Policies-final.pdf.

[28] LEY 33/2011, de 4 de octubre, General de Salud Pública. Madrid, Boletín Oficial del Estado, Miércoles 5 de octubre de 2011:104593-626. Recuperado en noviembre 2018 en https://www.boe.es/buscar/pdf/2011/BOE-A-2011-15623-consolidado.pdf.

[29] LINELL, A.; RICHARDSON, M. X. y WAMALA, S. (2013). «The Swedish national public health policy report 2010». Scandinavian Journal of Public Health, 41, 10, 3-56.

[30] MENEU, R. (2009). «Evaluación del buen gobierno sanitario. Algunas reflexiones para su mejora». En V. Ortún V. (dir.), El buen gobierno sanitario. Madrid, Springer Healthcare Communications, 24.

[31] MENEU, R. y ORTÚN, V. (2011). «Transparencia y buen gobierno en sanidad. También para salir de la crisis». Gaceta Sanitaria, 25, 4, 333-338. Recuperado en noviembre 2018 en http://www.econ.upf.edu/ ortun/publicacions/GacSanit2011 .pdf.

[32] MENEU, R. (2016). «Para priorizar es prioritario. Notas para deliberar sobre el establecimiento de prioridades en políticas de salud». En I. Hernández Aguado (coord.), Definición de prioridades en las políticas de salud. Barcelona, Fundación Dr. Antonio Esteve, 36, 3-9. Recuperado en noviembre 2018 de https://www.esteve.org/capitulos/ documento-completo-11/.

[33] MENEU, R. y ORTÚN, V. (2014). «Del buen gobierno y nuestras instituciones». Economía y Salud. Boletín Informativo, 80. Recuperado en noviembre 2018 de http://www. aes.es/boletines/news.php?idB=21\&idN=1312. 
[34] MILLER, D. y HERKINS, C. (2010). «Corporate strategy, corporate capture: Food and alcohol industry lobbying and public health». Critical Social Policy, 30, 564-589.

[35] MOODIE, R.; STUCKLER, D. y MONTEIRO, C. et al. (2013). «Profits and pandemics: prevention of harmful effects of tobacco, alcohol, and ultra-processed food and drink industries». Lancet, 381, 9867, 670-679.

[36] OECD. (2017). Health at a Glance. OECD Indicators. Paris, OECD.

[37] OLIVA, J.; PEIRÓ S. y PUIG-JUNOY, J. (2013). «Sistema Nacional de Salud y propuesta de reformas: más allá del ruido y la furia». Derecho y Salud, 23, 6-18. Recuperado en noviembre 2018 de http://www.econ.upf.edu/ puig/publicacions/Any\%202013/ DyS\%202013\%200liva.pdf.

[38] ORGANIZACIÓN MUNDIAL DE LA SALUD (OMS). (2001). Informe sobre la salud en el mundo 2000 - Mejorar el desempeño de los sistemas de salud. Ginebra, OMS. Recuperado en noviembre 2018 en http://apps.who.int/iris/bitstream/handle/10665/42357/ WHR_2000_spa.pdf? sequence $=1 \&$ isAllowed $=y$.

[39] ORTÚN, V. (2007). «A la política rogando y con el mazo dando (en los servicios sanitarios». Gaceta Sanitaria, 21, 6, 486-489. Recuperado en noviembre 2018 en https:// repositori.upf.edu/bitstream/handle/10230/32222/Ortun_GacetaSanitaria_salu.pdf?sequence $=1 \&$ is Allowed $=y$.

[40] ORTÚN-RUBIO, V. (2009). «Introducción». En V. ORTÚN-RUBIO, M. CALLEJÓN, J. COLOMER, J. GERVAS y R. MENEU R. et al. (eds.), El buen gobierno sanitario. Madrid, Springer.

[41] ORTÚN, V. (2018). «Priorizar o salir del paso». En Prioridades y políticas sanitarias. Barcelona, Fundació Víctor Grífols i Lucas. Cuadernos de la Fundació Víctor Grífols i Lucas, 48, 29-40.

[42] PEIRÓ, V.; ARTELLS, J. J. y MENEU, R. (2011). «Identificación y priorización de actuaciones de mejora de la eficiencia en el Sistema Nacional de Salud». Gaceta Sanitaria, 2, 95-105. Recuperado en noviembre 2018 de http://apps.elsevier.es/watermark/ctl_servlet?_f=10\&pident_articulo $=90003170 \&$ pident_usuario $=0 \&$ pcontac $t i d=\&$ pident_revista $=138 \& t y=56 \&$ accion $=L \&$ origen $=$ elsevier $\& w e b=w w w . e l$ sevier. es\&lan $=$ es\&fichero $=138 v 25$ n02a90003170pdf001.pdf.

[43] RAJMIL, L.; TAYLOR-ROBINSON, D.; GUNNLAUGSSON, G.; HJERN, A. y SPENCER, N. (2018). «Trends in social determinants of child health and perinatal outcomes in European countries 2005-2015 by level of austerity imposed by governments: a repeat cross-sectional analysis of routinely available data». BMJ Open, 8, e022932.

[44] REPULLO LABRADOR, J. R. (2012a). «La sostenibilidad de las prestaciones sanitarias públicas». En M. A. Presno Linera (coord.), Crisis económica y atención a las personas y grupos vulnerables. Oviedo, Procura n. ${ }^{\circ}$ 2, Procuradora General del Principado de Asturias, Universidad de Oviedo. Recuperado en noviembre 2018 de https://presnolinera.files.wordpress.com/2013/09/crisis-econc3b3mica-y-atencic3b3n-a-las-personas-y-grupos-vulnerables.pdf.

[45] REPULLO LABRADOR, J. R. e INFANTE CAMPOS, A. (2013). «La cohesión del SNS y su sostenibilidad: en el aniversario de una Ley que buscó de forma tardía e insuficiente gobernar un sistema descentralizado». Derecho y Salud, 23 (extraordinario), 83-95 . Recuperado en noviembre 2018 de https://www.ajs.es/revista-derecho-y-salud/ la-cohesion-del-sns-y-su-sostenibilidad-en-el-aniversario-de-una-ley-que-busco-deforma-tardia-e-ins. 
[46] REPULLO, J. R. y FREIRE, J. M. (2016). «Implementando estrategias para mejorar el gobierno institucional del Sistema Nacional de Salud». Gaceta Sanitaria, 30 (S1), 3-8.

[47] SANTANA, P. et al. (2017). Atlas of population health in European Union regions. Coimbra: Universidade de Coimbra.

[48] SOCIEDAD ESPAÑOLA DE SALUD PÚBLICA Y ADMINISTRACIÓN SANITARIA (SESPAS) (2015). Posicionamiento SESPAS en el año electoral 2015: Protege tu salud con tu voto. Barcelona, SESPAS. Recuperado en noviembre 2018 de http://sespas. es/wp-content/uploads/2017/01/SESPAS-Elecciones-2015.pdf.pdf.

[49] STIGLITZ, J. E.; SEN, A. y FITOUSSI, J.-P. (2009). «Report by the Commission on the Measurement of Economic Performance and Social Progress.

[50] SUBIRATS, J. (2001). «El análisis de las políticas públicas». Gaceta Sanitaria, 15, 259-64. Recuperado en octubre 2018 de http://www.gacetasanitaria.org/espdf-S0213911101715579.

[51] THE GUARDIAN (2018). «Fruit, veg and family life-why Spaniards are living longer». The Guardian, 19 de octubre. Recuperado en octubre 2018 de https://www.theguardian.com/world/2018/oct/19/spaniards-living-longer-researchers-credit-diet-lifestyle-fruit-veg-family-life.

[52] THE TIMES (2018). «They drink, they smoke, so why are the Spanish living so long?». The Times, 20 de octubre. Recuperado en octubre 2018 de https://www.thetimes.co.uk/ article/they-drink-they-smoke-so-why-are-the-spanish-living-so-long-tg7jzm0tb.

[53] VAN DEN HOOGEN, P. C.; FESKENS, E. J.; NAGELKERKE, N. J.; MENOTTI, A.; NISSINEN, A. y KROMHOUT, D. (2000). «The relation between blood pressure and mortality due to coronary heart disease among men in different parts of the world. Seven Countries Study Research Group». New England Journal of Medicine, 342, 1-8.

[54] VILLORIA MENDIETA, M. (dir.) (2014). Una evaluación del lobby en España: análisis y propuestas. Madrid, Transparency International España. https://webantigua.transparencia.org.es/estudio_lobby/spain_lobbying_esp_final.pdf.

[55] WORLD HEALTH ORGANIZATION (2008). Health Systems Governance: Toolkit on Monitoring Health Systems Strengthening. Geneva: WHO.

[56] ZARAGOZA GAYNOR, G.; HERNÁNDEZ-AGUADO, I. y PARKER L. A. (2016). «Colaboraciones público-privadas en salud pública: ¿parte de la solución o parte del problema?». Gestión Clínica Sanitaria, 18, 3, 75-7. Recuperado en noviembre 2018 de http://www.iiss.es/gcs/gestion63.pdf. 